\title{
Abnormal myocardial repolarisation in response to hypoxaemia and fenoterol
}

\author{
David G Kiely, Robert I Cargill, Alison Grove, Allan D Struthers, Brian J Lipworth
}

\begin{abstract}
Background - Prolongation of the QTc interval has been associated with cardiac dysrhythmias and sudden death. QTc dispersion (interlead variability in QTc interval) has recently been proposed as being a more sensitive marker of repolarisation abnormalities and shown to be a more specific index of arrhythmia risk. Although hypoxaemia and fenoterol have previously been shown to prolong the QTc interval, this does not reflect regional myocardial repolarisation abnormalities.

Methods - Electrophysiological effects were measured at baseline and after 30 minutes steady state hypoxaemia at an arterial oxygen saturation $\left(\mathrm{SaO}_{2}\right)$ of $75-$ $80 \%$ (study 1) and at baseline then $30 \mathrm{~min}$ utes after inhaled fenoterol $2.4 \mathrm{mg}$ (study 2). From the ECG, lead II corrected QT interval (QTc) and overall corrected QT dispersion were measured using a computer linked digitising tablet according to standard criteria.
\end{abstract}

Results - QTc dispersion was increased during hypoxia compared with baseline values (mean (SE) 69 (6) ms $v 50$ (5) ms) and after fenoterol compared with baseline (79 (13) $v 46$ (4) ms), respectively. There was also an increase in QTc interval and heart rate after fenoterol (493 (23) $v 420$ (6) ms and 98 (3) $v 71$ (6) bpm, respectively). The heart rate was increased during hypoxaemia compared with baseline (78 (3) $v 64$ (2) bpm), but no change occurred in the QTc interval.

Conclusions - Both hypoxaemia and fenoterol cause myocardial repolarisation abnormalities in man in terms of increased QTc dispersion, but only fenoterol increased the QTc interval. This may be relevant in the aetiology of arrhythmias in patients with acute severe asthma where $\beta$ agonist therapy and hypoxaemia coexist. (Thorax 1995;50:1062-1066)

Keywords: hypoxaemia, $\beta$ agonist, QT dispersion.

The surface electrocardiogram has been investigated for its ability to identify those patients at risk of arrhythmias and as such represents a cheap, non-invasive and simple method. Traditionally the single measurement of QTc interval has been used to measure the recovery of ventricular excitability, widely recognised to be the most important factor in arrhythmogenesis.

Prolongation of the QT interval has been shown to be a predictor of sudden death in alcoholic cirrhosis, ${ }^{1}$ sudden cardiovascular mortality in apparently healthy individuals, ${ }^{2}$ as well as sudden death in patients with ischaemic heart disease. ${ }^{3}$ Recently, however, QTc dispersion (interlead variability in QTc interval) has been suggested as a more sensitive and specific marker as it measures differences in regional repolarisation and has been shown to be increased in patients with hypertrophic obstructive cardiomyography (HOCM) at risk from ventricular arrhythmias, ${ }^{4}$ and in patients with long QT intervals it distinguished between those with ventricular arrhythmias and those without. ${ }^{5}$ It has also been found to be a more sensitive and specific marker of sudden death in heart failure than QTc interval alone. ${ }^{6}$

Cardiac arrhythmias are a very common finding in patients with respiratory failure and chronic obstructive pulmonary disease (COPD). ${ }^{7-9}$ It has been shown that depressed left ventricular diastolic performance is a predictive factor for ventricular arrhythmias during respiratory failure from COPD, although the poor definition of the statistical model suggests that other factors contribute to the genesis of these arrhythmias. ${ }^{10}$ In this respect Stewart et $a l^{11}$ have recently shown that QTc prolongation is a better predictor of death in patients with COPD than hypoxaemia, hypercapnia, or spirometric measurements. Interest has also surrounded the use of $\beta$ agonists which have inotropic, chronotropic, and electrophysiological effects. In particular, the epidemiological observation that fenoterol was associated with an increased risk of death in patients with severe asthma ${ }^{1213}$ has suggested that this drug may predispose these individuals to arrhythmias. Interestingly, the use of high dose nebulised $\beta$ agonists has been associated with an increased risk of cardiac arrhythmias in patients with COPD, ${ }^{14}$ and a further study revealed that normoxaemic patients with COPD had a higher frequency of ventricular ectopy during treatment with high dose than with low dose terbutaline, although 24 hour Holter recordings did not reveal an increase in significant arrhythmias. ${ }^{15}$

We have therefore, for the first time, looked at the effects of two separate stimuli which have been suggested as important factors in arrhythmogenesis in respiratory disease - 
namely, hypoxia and $\beta$ agonists - and their relative effects on QTc dispersion and QTc interval.

\section{Methods}

SUBJECTS

Sixteen healthy male volunteers aged 21-37 years were studied. There was no abnormality present on clinical history, clinical examination, 12 lead ECG, biochemical, or haematological screening. Patients abstained from alcohol and caffeine for a 24 hour period prior to the study. Informed written consent to the study protocol, previously approved by the Tayside committee for medical research ethics, was obtained.

\section{STUDY PROTOCOL}

Study 1

Eight subjects were studied. An intravenous cannula was inserted into the left forearm for blood sampling after which they rested supine for at least 30 minutes to obtain stable resting haemodynamics $\left(\mathrm{T}_{0}\right)$. They were then rendered hypoxaemic for 30 minutes by breathing a variable mixture of oxygen and nitrogen which rendered their arterial oxygen saturation between $75 \%$ and $80 \%\left(T_{1}\right)$. The hypoxic gas mixture was produced from separate cylinders of nitrogen and oxygen fitted with variable flow valves. Gases were mixed in a 25 litre Douglas bag, from which the subjects breathed through a mouthpiece connected by a series of one way valves while wearing an occlusive nose clip. A 12 lead ECG and venous blood samples for catecholamine and serum potassium assays were taken at $T_{0}$ and $T_{1}$.

\section{Study 2}

Eight subjects were studied. An intravenous cannula was inserted into the left forearm for blood sampling after which they rested supine for at least 30 minutes to obtain stable resting haemodyamics $\left(\mathrm{T}_{0}\right)$. Patients were then given fenoterol $2.4 \mathrm{mg}$ from a metered dose inhaler (MDI) via a spacer device $(12 \times 0.2 \mathrm{mg}$ actuations with three inhalations per actuation). A 12 lead ECG and venous blood samples for serum potassium were taken at $T_{0}$ and 30 minutes after inhalation of fenoterol $\left(T_{1}\right)$.

\section{MEASUREMENTS}

\section{OT interval}

The ECGs from both study days were analysed in random order after completion of the study by an investigator who was not involved during either of the study days and who was blinded with respect to the stimulus the volunteers had received. If feasible, the QT interval was measured in all leads of a surface 12 lead ECG (paper speed $=25 \mathrm{~mm} / \mathrm{s}$ ). Three consecutive cycles were measured in each lead where possible and the mean value was taken as representing the QT interval in that lead. The QT interval was calculated according to standard
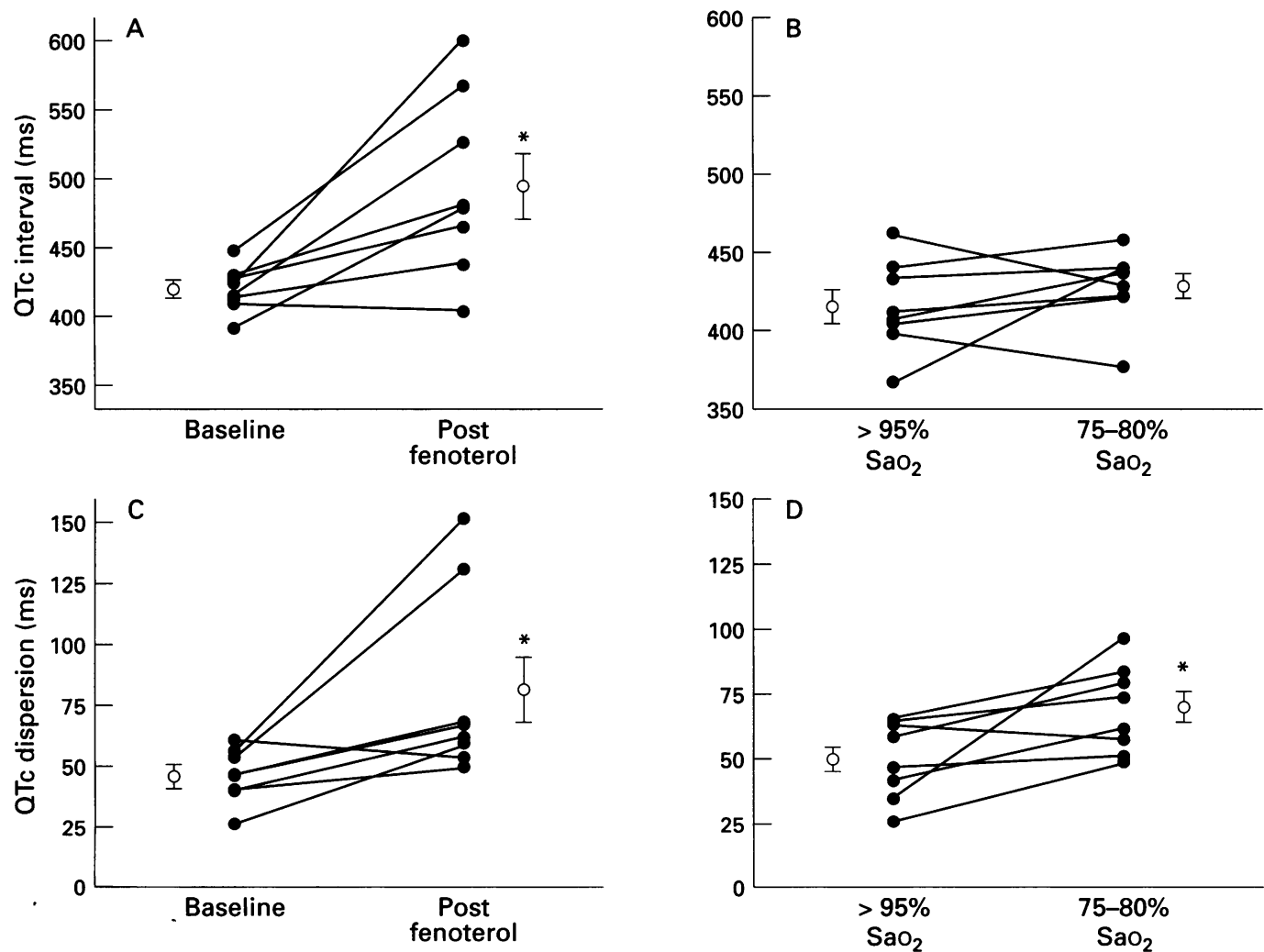

Figure 1 Effects of hypoxaemia and fenoterol on QTc interval and QTc dispersion. (A) Absolute QTc interval measured at baseline and after fenoterol; ${ }^{*} p<0.01$ fenoterol treatment versus baseline. (B) Absolute QTc interval measured at baseline and during hypoxaemia. (C) Absolute OTc dispersion measured at baseline and after fenoternl; ${ }^{*} p<0.05$ fenoterol treatment versus baseline. (D) Absolute $Q T c$ dispersion measured at baseline and during hypoxaemia; ${ }^{*} p<0.05$ fenoterol treatment versus baseline. (D) Absolute $Q T c$ dispersion measured at baseline and durng hypoxaemia;
${ }^{*} p<0.05$ fenoterol treatment versus baseline. Values for the same patient are connected and the mean and standard error of the mean are represented as clear circles and error bars, respectively. 
criteria $^{16}$ from the onset of the QRS complex to the end of the $T$ wave - that is, a return to the $T / P$ baseline. In the presence of $U$ waves the QT interval was measured to the nadir of the curve between the $\mathrm{T}$ and the $\mathrm{U}$ waves. QT intervals were then corrected for rate using Bazett's formula (QTc $=\mathrm{QT} / \sqrt{ } \mathrm{RR}) .^{17}$

QTc dispersion was defined as the difference between the maximum and minimum QTc interval measured during analysis of all leads of the surface ECG. ${ }^{16}$ The measurements were made using a computer linked digitising tablet. ${ }^{18}$ To compare the standard measure of QTc interval with QTc dispersion we calculated the average of three QT intervals in lead II and corrected for heart rate using Bazett's formula $(\mathrm{QTc}=\mathrm{QT} / \sqrt{ } \mathrm{RR})$.

\section{Heart rate}

This was calculated from five consecutive $R$ to $\mathrm{R}$ intervals in lead II.

\section{Oxygenation}

Arterial blood oxygen was continuously monitored by transcutaneous oximetry (CSI 503, Criticare Systems Inc, Waukesha, Wisconsin, USA).

\section{Serum potassium}

Samples for serum potassium were collected in chilled lithium-heparin tubes and centrifuged at $4^{\circ} \mathrm{C}$ immediately. The separated plasma was stored at $-20^{\circ} \mathrm{C}$ until measured in one batch at the end of the study using an internal caesium standard flame photometer (Instrumentation Laboratory, Milan, Italy).

\section{Catecholamines}

Samples for adrenaline and noradrenaline were collected in chilled lithium-heparin tubes and centrifuged immediately at $4^{\circ} \mathrm{C}$. Separated plasma was stored at $-70^{\circ} \mathrm{C}$ and assayed in one batch at the end of the study using the double isotope radioenzymatic method. ${ }^{19}$

\section{DATA ANALYSIS}

Comparison of values between points was made by multifactorial analysis of variance (MANOVA). ${ }^{20}$ A probability value of $p<0.05$ (two tailed) was considered to be statistically significant. Data are presented as means and the standard error of the mean (SE).

\section{Results}

QTc interval

Treatment with fenoterol significantly $(\mathrm{p}<0.01)$ increased QTc interval compared with baseline (493 (23) v 420 (6) ms; fig 1A). Hypoxaemia, however, had no significant effect (fig. 1B).

\section{QTc dispersion}

Fenoterol significantly $(\mathrm{p}<0.05)$ increased QTc dispersion compared with baseline $(79$ (13) $v$
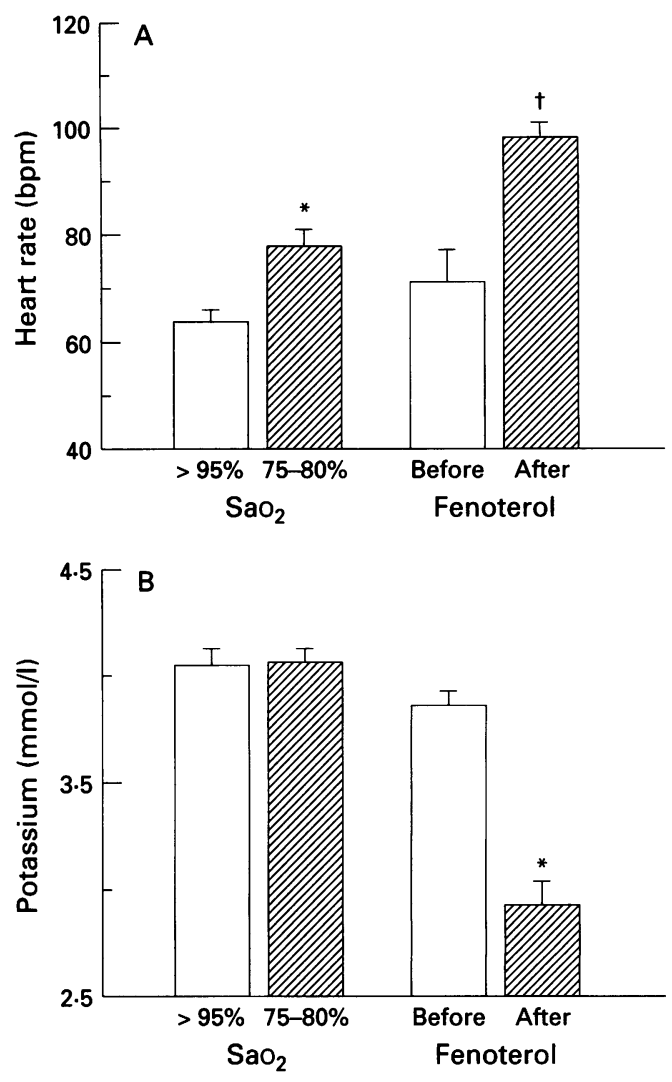

Figure 2 Effects of hypoxaemia and fenoterol on heart rate and serum potassium. (A) Left: absolute heart rate

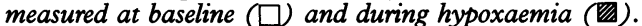
Right: absolute heart rate measured at baseline ( $\square$ ) and after fenoterol $(\mathbb{Z})$. ${ }^{*} p<0.05$ difference between hypoxaemia and baseline; $\dagger p<0.01$ difference between fenoterol and baseline. (B) Left: serum potassium measured at baseline ( $\square$ ) and during hypoxaemia (田). Right: serum potassium measured at baseline ( $\square$ ) and after fenoterol $(\mathbb{Z})$. ${ }^{*} p<0.0001$ difference between fenoterol and baseline.

46 (4) ms; fig 1C). Similarly, hypoxaemia significantly $(p<0.05)$ increased QTc dispersion compared with baseline (69(6) $v 50$ (5) ms; fig 1D).

\section{Heart rate}

The heart rate was significantly increased by both fenoterol (98(3) $v 71$ (6) bpm; p<0.01) and hypoxaemia (78 (3) $v 64$ (2) bpm; $\mathrm{p}<0.05)$ compared with baseline values (fig $2 \mathrm{~A}$ ).

\section{Serum potassium}

Treatment with fenoterol resulted in a significant $(p<0.0001)$ reduction in serum potassium compared with baseline values $(2.93(0.12)$ v $3.86(0.07) \mathrm{mmol} / \mathrm{l})$. Hypoxaemia, however, had no significant effect (fig 2B).

\section{Catecholamines}

Hypoxaemia had no significant effect on either noradrenaline $(4.75(0.65) v 3.97(0.36) \mathrm{nmol} / \mathrm{l})$ or adrenaline $(0.22(0.02) v 0.16(0.02) \mathrm{nmol} /$ 1) compared with baseline values. 


\section{Discussion}

Our results show that both hypoxaemia and fenoterol significantly increase QTc dispersion in healthy volunteers, suggesting that these two stimuli cause abnormalities in myocardial repolarisation. However, only fenoterol significantly increased QTc interval and significantly decreased the serum potassium concentration compared with baseline changes which have previously been documented in normal and asthmatic subjects. ${ }^{2122}$

It is thought that QTc dispersion reflects regional variation in ventricular repolarisation and existing evidence suggests that it is a powerful index of the propensity for developing life threatening arrhythmias. ${ }^{4-6}$ The underlying mechanism responsible for an increase in QTc dispersion is not known, although it has been suggested that fibrosis may be important. ${ }^{6} \mathrm{How}-$ ever, as our results suggest, dynamic physiological abnormalities must play a part as both stimuli are acute and self limiting in nature. Indeed, our results are not the first to implicate dynamic variables as having an important role in altering QTc dispersion; Moreno et $a l^{23}$ have shown that QTc dispersion is decreased following successful thrombolysis after myocardial infarction, demonstrating that localised ischaemia affects regional repolarisation. In the context of congestive cardiac failure no correlation was noted between increased QTc dispersion and catecholamine levels. ${ }^{6}$ We were unable to show any increase in circulating catecholamines during acute hypoxia, although this was a small study and as such has an inherently large type 2 error. It is known that large oral doses of $\beta$ agonists do not affect catecholamine levels; ${ }^{24}$ they were not measured in this study although Scheinin et al have shown that noradrenaline was dose dependently increased by fenoterol treatment. ${ }^{25}$ It may be that hypoxia and $\beta$ agonists have their effects on QTc dispersion by altering autonomic tone, although it is likely that local metabolic and electrical disturbances may play a part. In this respect, hypokalaemia affects the electrical stability of cell membranes and is known to predispose individuals to arrhythmias and may explain, at least in part, why fenoterol increases QTc dispersion.

Although hypoxia did not significantly increase QTc interval in this study, this phenomenon has previously been documented. ${ }^{21}$ However, it may be that QTc dispersion is a more sensitive marker of abnormal myocardial repolarisation than QTc interval alone.

With respect to our methodology there are undoubtedly limitations in all studies measuring QTc dispersion. We used a computer linked digitising tablet which has been shown by other investigators to be a reliable and accurate measure of QTc dispersion. ${ }^{18}$ Probably the most important aspect concerning methodology is the protocol to define the end of the $T$ wave. We have thus used the most commonly used protocol ${ }^{16}$ and one which has been shown to correlate with risk of arrhythmia and sudden death. We also measured the QTc interval in the majority of leads in each individual, although occasionally leads were omitted due to difficulty defining the end of the $T$ wave. We used routine
ECGs to measure QTc dispersion since we felt this would have most clinical relevance and, indeed, no substantial evidence suggests that simultaneous ECG recording has any benefits.

What is the clinical relevance of QTc dispersion and what, if any, are its merits compared with our traditional measure of ventricular repolarisation, QTc interval? Firstly, much time and effort has been expended to find markers of mortality in hypoxic chronic lung disease and to find high risk patients who may benefit from further investigation or treatment. It would certainly be of interest if QTc dispersion correlates with risk of sudden death in patients with cor pulmonale. The use of fenoterol has been associated with an increased incidence of asthma deaths. Could it be possible that this observation is due to arrhythmias occurring in the presence of abnormal myocardial repolarisation? It has been suggested that certain individuals may be more susceptible to the effects of $\beta$ agonists. In this respect it is interesting to note the large increase in QTc dispersion that occurred in two individuals in response to inhaled fenoterol (fig 1C). Although we exceeded the standard dose of fenoterol suggested by the manufacturer, in an asthmatic attack very high doses of $\beta$ agonists may have to be taken ${ }^{26}$ and, indeed, the British Thoracic Society guidelines recommend up to 50 puffs of a $\beta$ agonist from a metered dose inhaler during a severe attack. ${ }^{27}$ We have also shown that acute hypoxaemia increases QTc dispersion suggesting that this may have a part to play in arrhythmogenesis in acute asthma, although we can infer little concerning chronic hypoxia. Compared with the QTc interval, QTc dispersion, which measures ventricular repolarisation in several leads, may be a better indicator of arrhythmia risk. Indeed, it has been shown to be both a more sensitive and specific marker of sudden death than QTc interval alone. ${ }^{6}$ Amiodarone which is known to prolong QTc interval is used in the treatment of ventricular tachycardia. It is perhaps no coincidence that this drug also reduces QTc dispersion. ${ }^{28}$

Thus, we have shown for the first time that two stimuli thought to be associated with arrhythmogenesis in respiratory disease increase QTc dispersion - namely, hypoxia and $\beta$ agonists. It seems likely that this index of abnormal myocardial repolarisation will have important implications in terms of risk stratification of patients and in the development of new drugs and the investigation of current drugs in respiratory medicine.

1 Day CP, James OFW, Butler TJ, Campbell RWF. QT interval prolongation and sudden cardiac death in patients with alcoholic liver disease. Lancet 1993;341:1423-8.

2 Schouten EG, Dekker JM, Meppelink P, Kok FJ, Vandenbroucke JP, Pool J. QT interval prolongation predicts

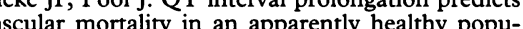
lation. Circulation 1991;84:1516-23.

3 Puddu PE, Bourassa MG. Prediction of sudden death from QTc interval prolongation in patients with chronic ischQTc interval prolongation in patients with chronic isch

4 Buja G, Miorelli M, Turrini P, Melacini P, Nava A. Comparison of QT dispersion in hypertrophic cardiomyopathy parison of QT dispersion in hypertrophic cardiomyopathy between patients with and without ventricular arrh

5 Day CP, McComb JM, Campbell RWF. QT dispersion: an indication of arrhythmia risk in patients with long QT indication of arrhythmia risk in pa
intervals. Br Heart $\mathcal{f} 1990 ; 63: 342-4$

6 Barr CS, Naas A, Freeman M, Lang CC, Struthers AD. QT dispersion and sudden unexpected death in chronic heart failure. Lancet 1994;343:327-9. 
7 Terlapur VG, Mir NA. Nocturnal hypoxaemia and associated electrocardiographic changes in patients with chronic obstructive airways disease. $N$ Engl 7 Med 1982; 306: 125-30.

8 Holford FD, Mithoefer JC. Cardiac arrhythmias in hospitalized patients with chronic obstructive pulmonary disease. Am Rev Respir Dis 1973;108:879-85.

9 Kleiger RE, Senior RM. Longterm electrocardiographic monitoring of ambulatory patients with chronic airway monitoring of ambulatory patien
obstruction. Chest 1974;65:483-7.

10 Raffaele AI, Riccardo P, Leonello F, Cocchi A, Massimo GB, Alessandro G. Cardiac arrhythmias and left ventricular function in respiratory failure from chronic obstructive pulmonary disease. Chest 1990;97:1092-7.

11 Stewart AG, Waterhouse JC, Howard P. The QTc interval, autonomic neuropathy and mortality in hypoxaemic COPD. Respir Med 1995;89:79-84.

12 Crane F, Pearce NE, Flatt A, Burgess C, Jackson R, Kwong $\mathrm{T}$, et al. Prescribed fenoterol and death from asthma in New Zealand, 1981-83. Case controlled study. Lancet 1989;i:917-22.

13 Grainger J, Woodman K, Pearce N, Crane J, Burgess C, Keane A, et al. Prescribed fenoterol and death from asthma in New Zealand. 1981-87: a further case-control study. in New Zealand. 1981-87.

14 Higgins RM, Cookson WOCM, Lane DG, John SM, McCarthy GL, McCarthy ST. Cardiac arrhythmias caused by nebulised beta-agonist therapy. Lancet 1987; ii:863-4.

15 Lipworth BJ, Clark RA, Dhillon DP, McDevitt DG. Comparison of the effects of prolonged treatment with low and high doses of inhaled terbutaline on beta-adrenoreceptor responsiveness in patients with chronic obstructive pulmonary disease. Am Rev Respir Dis 1990;142:338-42.

16 Higham PD, Campbell RWF. QT dispersion. $B r$ Heart 7 1994;71:508-10.

17 Bazett HC. An analysis of the time relations of the electrocardiogram. Heart 1920;7:353-70.

18 Bhullar HK, Fothergill JC, Goddard WP, de Bono DP.
Automated measurement of QT dispersion from hard copy ECGs. F Electrocardiol 1993;26:321-33.

19 Brown MJ, Jenner DA. Novel double isotope technique for enzymatic assay of catecholamines, permitting high precision, sensitivity and plasma sample capacity. Clin $S_{c i}$ 1980;61:591-5.

20 Brown RA, Beck IS. Comparison of several groups: The analysis of variance. In: Brown RA, Beck JS, eds. Medical statistics in microcomputers. London: British Medical Journal statistics in microcomputers.
Publishing, 1990:34-42.

21 Bremner P, Burgess CD, Crane J, McHaffie D, Galletly D, Pearce $\mathrm{N}$, et al. Cardiovascular effects of fenoterol under conditions of hypoxaemia. Thorax 1992;47:811-7.

22 Newnham DM, Wheeldon NM, Lipworth BJ, McDevit DG. Single dosing comparison of the relative cardiac $\beta_{1}$ $\beta_{2}$ activity of inhaled fenoterol and salbutamol in norma subjects. Thorax 1993;48:656-8.

23 Moreno FL, Villanueva T, Karagounis LA, Anderson JL Reduction in QT interval dispersion by successful thrombolytic therapy in acute myocardial infarction. Circulation 1994;90:94-100.

24 Barnes PJ. Adrenergic mechanisms in asthma. DM Thesis. Oxford: University of Oxford, 1982.

25 Scheinin M, Koulu M, Laurikainen E, Allonen H. Hypokalaemia and other non-bronchial effects of inhaled fenoterol and salbutamol: a placebo controlled dose response study in healthy volunteers. Br f Clin Pharmacol 1987;24: 645-53.

26 Windom $\mathrm{H}$, Burgess $\mathrm{C}$, Crane J, Pearce N, Kwong T, Beasley $R$. The self administration of inhaled beta agonist drugs during severe asthma. NZ Med f 1990;103:205-7.

27 British Thoracic Society. Guidelines for management of asthma in adults. II - Acute severe asthma. BMF 1990; 301:797-800.

28 Dritsas A, Gilligan D, Nihoyannopoulos P, Oakley CM. Amiodarone reduces $\mathrm{QT}$ dispersion in patients with hypertrophic cardiomyopathy. Int $\mathcal{f}$ Cardiol 1992;36: 345-9. 